\title{
EDITORIAL
}

\section{ON The Growth OF ThIS JOURnAL}

The excitement of the inauguration of this journal is hardly over and we are now presenting the next issue. There is a $38 \%$ increase in the number of papers. We are projecting more growth following it.

In this issue there are papers on resolution enhancement techniques, characterization of linewidth variation, imaging with laser ablation, electron beam fabrication of optical probes, electron projection lithography, magnetic microactuators, organic micromachining, silicon microsuspensions, silicon capillaries, and silicon diaphragms.

The most unique paper in this issue is the translation from German of the classic article by Fritz Zernike published 68 years ago in the Dutch journal Physica, on diffraction theory containing the introduction of the Zernike polynomials. These polynomials describe the aberration of imaging systems in cylindrical coordinates and have a unique orthogonal property that makes them the universal choice of analytic tool to define and to optimize diffraction-limited large-field high-NA lenses. Translating classic papers such as this into English and republishing in $\mathrm{JM}^{3}$ make the classics accessible to readers in the $M^{3}$ field. Occasionally, we will publish such articles if the original printing is very difficult to find or in a less familiar language, the new printing or translation is useful for readers of $\mathrm{JM}^{3}$, the work is well quoted, well respected, well established, and copyright is properly handled.

In the inaugural issue, there were papers on mask design, imaging, birefringence, resolution enhancement, anti-reflection coatings, thin film resonators, and micromirrors. For that issue there were more submissions in microlithography than in microfabrication or microsystems. In this issue, the three fields are well balanced. Actually, the synergy of the three fields makes it not so easy to differentiate the papers. For example, "Electron beam lithography of microbowtie structures for next-generation optical probe" may be designated as Microlithography and Microfabrication; ditto for "KrF excimer laser trenching of $\mathrm{X}$-cut $\mathrm{LiNbO}_{3}$ for realization of optimized optical modulator electrode structures." It is even harder to differentiate Microfabrication from Microsystems. I tend to put papers directly related to fabrication under Microfabrication and those related to integration, packaging, characterization, and those present- ing a wider perspective than just fabrication to Microsystems. However, in view of the synergy, distinct classification of the three fields is not absolutely necessary.

There are 10 contributions from academia and just 1 from industry. In the inaugural issue, the count was 2 and 6 , respectively. The pendulum has swung back and forth and likely will continue to swing. We have been reviewing our articles based on their usefulness and soundness regardless of the contributing institution and will continue to do so. Taking the two-issue total, the distribution of the contributing institutions reflects that academia is slightly more diligent at its pen as well as its work. Our colleagues in industry tend to be more concerned with the latter. We would like to encourage you to take an extra step to immortalize your hard work.

We would like to place an equal emphasis on theoretical understanding, experimental confirmation, relevant applications, and noteworthy innovations. The journal is aiming at being useful to readers in the microlithography, mask technology, micromachining, micromanufacturing, microelectronic manufacturing, smart structures, MEMS, MOEMS, and optoelecronics fields.

The journal may not look like the proceedings, because the articles are inevitably more rigorous and more carefully prepared. They have survived critical reviews and are written with archival publication in mind. The end product is that you will be proud of your work. As a reader and a potential author, you are an important ingredient in shaping the character of this journal.

There will be a special section on "Lithography for sub-100-nm devices" in the October issue this year, followed by one on "Sacrificial surface micromachining" in April 2003.You are encouraged to contribute to this latter special section. Please submit your papers before September I, 2002. Details are given in the Call for Papers in this issue.

Happy reading!

\section{Burn J. Lin Editor-in-Chief}

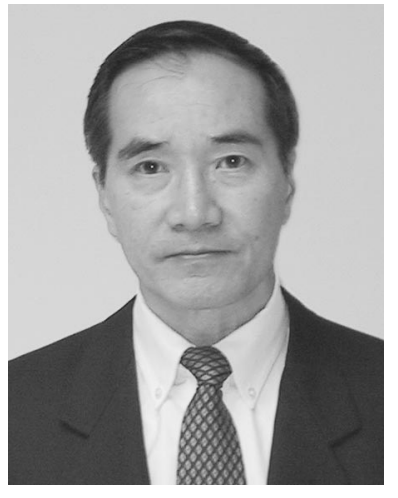

